$\underline{\text { Research Articles }}$

\title{
Resilience and adjustment trajectories amongst children in displacement-affected communities in Zarqa, Jordan
}

\author{
Sabrina Hermosilla ${ }^{1}$, Janna Metzler ${ }^{2}$, Kevin Savage ${ }^{3}$, Alastair Ager ${ }^{4}$ \\ ${ }^{1}$ Institute for Social Research, University of Michigan, Ann Arbor, Michigan, USA, 2 Department of Population and Family Health, Mailman School of \\ Public Health, Columbia University, New York City, New York, USA, ${ }^{3}$ Humanitarian and Emergency Affairs, World Vision International, Geneva, \\ Switzerland, ${ }^{4}$ Department of Population and Family Health, Mailman School of Public Health, Columbia University, New York City, New York, USA; \\ Institute for Global Health and Development, Queen Margaret University, Edinburgh, Scotland, UK \\ Keywords: mental health, global health, program evaluation, refugee \\ https://doi.org/10.29392/001c.18233
}

\section{Journal of Global Health Reports}

Vol. 4, 2020

\section{Background}

The experiences of protracted conflict and displacement are clear threats to children's developmental progress. Understanding the factors that shape the trajectories of children's well-being and adjustment in such contexts is important for informing interventions.

\section{Methods}

We collected data at three time points from a sample of Syrian refugee and Jordanian children $(n=650)$ residing in Zarqa, Jordan who met eligibility criteria for humanitarian programming. We assessed primary outcomes of protection concerns, caregiver stress, mental health, and developmental assets at three time points: baseline (T1), three months later (T2), and fifteen months after baseline (T3).

\section{Results}

Over the fifteen-month study period (T1-T3) child protection concerns and mental health symptoms improved, caregiver stress remained constant, and developmental assets deteriorated. School attendance was independently associated with improvements in protection concerns $(\beta=-1.05, P=0.01)$, caregiver stress $(\beta=-0.66, P=0.02)$, and developmental assets ( $\beta=3.84, P=0.02$ ). Concern over lost livelihoods significantly predicted higher protection concerns $(\beta=4.08, P<0.001)$ and caregiver stress $(\beta=2.32$, $P<0.001)$. Attending child-focused programming did not significantly impact primary outcomes.

\section{Conclusions}

This study documents the capacity for adjustment and adaptation of children in the context of protracted displacement. The significant influences of attending school and concern over lost livelihoods on observed trajectories indicate the importance of addressing structural factors, such as education and employment, in supporting processes of resilience in these populations. Programmatic activities for children may secure valuable shorter-term impacts but here, as elsewhere, failed to impact outcomes longer-term.

Conflict and displacement are clear threats to children's mental health and developmental progress. By the end of 2018, 70.8 million people were displaced by armed conflict and violence, more than half were under the age of $18 .{ }^{1}$ Children are particularly vulnerable during emergency situations. $^{2-4}$ Humanitarian crises have become protracted, lasting decades; $78 \%$ of refugees in 2018 were in exile for over five years. ${ }^{1}$ Children forced to resettle across international borders are faced with shifts in family dynamics and are often exposed to separation, exploitation and forced labor, sexual violence and rape, physical abuse, challenges in navigating a new education system, and food insecurity. ${ }^{5-7}$ While at increased risk, the specific mental health and development trajectories (and thus corresponding needs) of children are still poorly understood. ${ }^{8}$

After eight years of conflict, these threats and increased vulnerability are dire for children affected by the Syrian crisis. Syrian refugees account for over a quarter of the world's 20 million refugees, with nearly 2.4 million children displaced in the neighboring countries of Jordan, Lebanon, Turkey, Iraq, and Egypt. ${ }^{1,9}$ Jordan is host to the $10^{\text {th }}$ largest population of refugees in the world equating to well over 
half of a million people, half of which are children.1,10,11 As most refugees within Jordan have settled within local host communities, ${ }^{9}$ the challenges, instability, and resultant deleterious health and developmental effects may influence both Syrian children and their Jordanian hosts, however their specific mechanism and trajectories are currently unclear. ${ }^{12}$ Threats to the longer-term development trajectories of children in these contexts have elevated child mental health and development to a central research, programmatic, and policy priority. $5,13,14$ Navigating early-life stressors - and associated instability - that are characteristic of displacement-affected communities can have severe and lasting effects on children's mental health, developmental progress, and wellbeing across the lifecourse. ${ }^{5,15-17}$ Experiencing social adversity during childhood is associated with longer-term developmental problems such as speech and language difficulties, learning and physical disorders, and emotional and behavioral problems. ${ }^{12,18-20}$ When these exposures are at a population level (e.g. displacement-affected communities), social adversities - and resultant deleterious outcomes - cluster, with the potential to impact communities, countries, and regions for a generation. ${ }^{19,21-23}$ As the Syrian conflict continues, the potential for an entire generation of children to be 'lost' in its wake has united diverse actors towards a coordinated regional initiative to ensure children have access to resources and essential interventions. ${ }^{24}$

The concept of resilience is a useful frame for children's experience in situations of extreme adversity and is increasingly adopted by humanitarian practitioners and donors to understand and articulate the nature and role of service provision within the broader socio-ecological system of support, care, and protection critical to the adjustment and wellbeing of children. ${ }^{25}$ With this framework, mental health and psychosocial support interventions frequently seek to strengthen adaptive capacities and assets linked to healthy developmental milestones for children, as well as provide resource support to the child's protective environment, comprised of their family, peers, school, and various social and institutional support structures. ${ }^{25}$ Resilience is thus not just the capacity of individuals to access critical resources that sustain wellbeing, but also the interaction and communication between groups and individuals at various socio-ecological levels. ${ }^{26}$ Utilizing resilience-based approaches provides a unique opportunity to examine the often complex and dynamic processes of resource acquisition and negotiation that occur during periods of displacement while simultaneously monitoring and holistically assessing these resource needs. ${ }^{26,27}$ As a nascent framework, the evidence-base for such interventions is lacking.

This study seeks to identify the factors that were predictive of better adjustment and adaption amongst displacement-affected children over an extended period. This included engagement in child-focused programming activities, schooling, etc., as well as broader demographic predictors. Understanding the factors that shape the trajectories of children's well-being and adjustment in such contexts is essential in guiding future interventions.

\section{METHODS}

\section{THE CURRENT STUDY}

This prospective longitudinal study maps the trajectories of Syrian refugee and Jordanian children in a Jordanian community heavily affected by displacement resulting from the Syria crisis. The sampling frame constitutes children registered in 2014 for program activities provided by the Islamic Charity Center - in partnership with World Vision Jordan - in the southwest section of the urban capital of the Zarqa Governorate, Az-Zarqa. The study tracks protection concerns, caregiver stresses, mental health and psychosocial wellbeing, and development outcomes for these children over a fifteen-month period to identify which factors predicted more favorable outcomes.

Primary socio-demographic factors measured to discern their potential influence on outcomes include age, gender, nationality, school enrollment, and household composition, size, and livelihoods.

Another factor considered was engagement in the program activities offered by the Islamic Charity Center in the early months of refugee settlement in the area. Detailed intervention information is available elsewhere; briefly, this programming was delivered in the form of establishment of a Child Friendly Space (CFS). ${ }^{28}$ The CFS intervention was offered three days per week for two hours a day separately to two age ranges ( 5 to 12 years and 13 to 17 years) for up to 12 weeks. Children participating in the CFS were exposed to various structured activities, such as drama, singing, handicrafts, drawing, games, puzzles, storytelling, and informational videos. Children also engaged in sessions on life skills, such as hygiene, community mapping, and the importance of volunteerism.

\section{STUDY DESIGN}

The study protocol was conducted with ethical approval from the Columbia University Medical Center (Reference AAAJ4352) and the Ministry of Social Development for the Hashemite Kingdom of Jordan. The research was conducted in collaboration with Columbia University Middle East Research Center, World Vision Jordan, the Islamic Charity Center, and the Family Guidance and Awareness Center. The study location was the first site with new programming of CFS during the research time period, as previously determined by the strategic priorities of implementing partners.

Survey instruments and consent were developed in English, adapted from Arabic-validated where available,${ }^{29}$ then translated into Arabic by a native speaking content-specialist and back translated into English. Informed consent was obtained in Arabic from all adult participants. Caregivers were asked to participate if their child (aged six to seventeen) was registered for the intervention as either a participant or a waitlist control member. Caregivers were interviewed if their child was aged six to nine at the time of interview. For children aged ten to seventeen, after the study team documented their caregiver's consent, children were approached and provided their assent and were interviewed directly. Outreach was conducted in the neighborhoods adjacent to the community center (populated by primarily lower income Jordanians; Syrian and some Iraqi and 


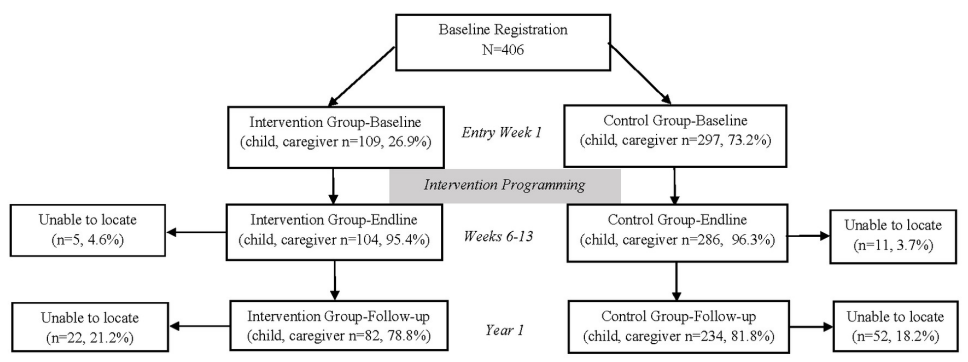

Figure 1. Study flow diagram.

Palestinian refugees) in the week preceding intervention registration. Children aged six to seventeen were eligible for intervention programming within the center. All families arriving during the registration window were provided with written or oral consent forms related to the evaluation interviews and asked to voluntarily participate in baseline interviews. ${ }^{28}$ Upon completion of the registration week, children were allocated into age-disaggregated activity sessions (intervention) on a first come, first serve basis; children not included in the first round of programming were placed on a waitlist for subsequent rounds of programminfg (waitlistcontrol).

Survey data collection took place in February 2014 (T1), June 2014 (T2), and from June to September 2015 (T3, initiating approximately one year after intervention completion, to estimate maintained long-term effects). The field team consisted of monitoring and evaluation specialists, graduate students, and a team of local enumerators. Interviews were conducted in semi-private locations in a large room within the community center at $\mathrm{T} 1$ and $\mathrm{T} 2$ and at a partner facility at T3. Participants were reimbursed for transportation at T3 in appreciation for their time, participation, and travel to the new partner facility, some distance from the original interview location (Figure 1).

\section{PRIMARY OUTCOMES}

Protection concerns. A question from the inter-agency Child Protection Rapid Assessment was adapted for use in Jordan $^{30}$ to assess protection concerns. Children and caregivers were asked which, if any, of these concerns about child protection were a source of stress since coming to Jordan (or since the recent Syrian migration flow to Jordan) or any others: 'not being able to go back to school', 'not be- ing able to return home', 'losing their home and belongings, 'being separated from their friends', 'being separated from their families (extended)', 'tension within the family (extended)', 'domestic violence', 'sexual violence', 'lack of jobs/ inability to provide an income for family', 'nightmares or bad memories', 'crowdedness within your living area', 'lack of safe play areas', 'lack of hope for their future.' Other child protection concerns were recorded as open-ended responses.

Caregiver stresses. A further question from the interagency Child Protection Rapid Assessment was adapted for assessment of caregiver stresses in this context. ${ }^{30}$ Children and caregivers were asked which, if any, of these stresses had been a concern for caregivers since coming to Jordan or any others: 'lack of food', 'not enough water', 'lack of shelter', 'lost property', 'lost livelihood', 'children's safety', 'lack of education', 'decreased access to healthcare.' Other sources of stress for caregivers were recorded as open-ended responses.

Mental health. The 21-item Arab Youth Mental Health scale $^{29}$ a screening tool for depression and anxiety in youth, was used to assess mental health. It includes questions such as 'During the last week I was bored and hated my life' and 'During the last week I was having a lot of headaches, stomach-aches, and nausea'. Items are rated on a scale: 1 = Rarely, 2 = Sometimes; 3 = Always, with higher scores indicative of more symptoms of anxiety and depression.

Developmental assets. The 13-item Emergency Developmental Assets Profile (EmDAP) ${ }^{31}$ assessed development assets within eight categories (e.g. positive identity, constructive use of time, social competencies) through child-completed items such as 'I feel optimistic about the future' and 'I think it is important to help people'. Items are rated on a 
scale: 0 = Rarely, 1 = Sometimes, 2 = Often, 3 = Almost Always, with higher scores indicative of a greater amount of assets essential for healthy developmental progress. Quartile ranges indicating Good, Adequate, Vulnerable, and Highly Vulnerable levels of developmental assets vary across cultures. A caregiver-reported developmental assets Caregiver Rating of Developmental Assets (CDRA) profile - mirroring items of the EmDAP - was administrated to caregivers to answer in relation to their perceptions of their child.

\section{ADDITIONAL MEASURES}

Socio-demographic variables. Individual items related to child age, gender, and nationality were asked at each time period (T1, T2, and T3). Household factors such as: vulnerability designation (developed by program team around standards for beneficiary reporting; included factors: greater than six in household, primary caregiver is only parent in household or has chronic disease, and child in household has disability or chronic disease), primary caregiver relation to children (e.g., biological mother, father, aunt, uncle, grandparent, etc.), nationality, household size, and primary livelihoods, were also collected at each time period (T1, T2, T3). ${ }^{28}$

Program activities. Children and caregivers were asked to report frequency of attendance for the CFS program (T1 and $\mathrm{T} 2$ ); for formal schooling (T1, T2 and T3); and for extra-curricular activities (T1, T2, and T3). CFS self-reported attendance was cross-referenced with facility-kept records to determine item reliability.

\section{STATISTICAL ANALYSIS}

After quantitative data were cleaned, univariate and bivariate analyses were conducted to describe the sample and explore key patterns by age group, gender, and CFS attendance status. Cronbach's alpha and Kuder-Richardson Formula 20 (KR20) were run to explore scale reliability by age and gender subgroups. Histograms and boxplots were created to explore quartile distribution of key outcome variables and normality distribution assumptions. Longitudinal generalized linear multivariable (GLM) models, controlling for design effects, were estimated based on important $(P<0.10)$ bivariate findings and variables of epidemiologic significance, by outcome and age group, to explore unique contribution of key factors to main outcomes. Model diagnostics, including testing for multicollinearity (through variance inflation factors) amongst final model variables, were completed on all GLM models. To estimate program effect, we calculated crude Cohen's $d$ for each study outcome (recoded when necessary so that a positive value indicates a salubrious effect). A Cohen's $d$ of 0.20 is considered a small effect, 0.50 a moderate effect, and 0.80 or above a large effect. ${ }^{32}$ Observations with missing data were pairwise deleted. All analyses were conducted in STATA 14.2 (StataCorp, College Station, TX).

\section{RESULTS}

\section{CHARACTERISTICS OF THE SAMPLE}

235 caregivers of young children aged 6 to 9 years were traced over time with 168 retained for data collection at $\mathrm{T} 2$ and 141 retained at T3. 179 children aged 10 to 12 years were traced throughout the study period with 120 retained at T2 and 108 retained at T3. 236 older children aged 13 to 18 were traced throughout the study period with 132 retained at $\mathrm{T} 2$ and 132 retained at $\mathrm{T} 3$ (see Figure 1 ). $99.6 \%$ of the sample was missing two or fewer analytic variables. The sample was comprised of 59.4\% Syrian children, 34.2\% Jordanian children, and 5.9\% Palestinian children (see Table 1). Children who reported at T2 to have attended CFS regularly (reported 'sometimes' or 'always' attended) were designated for analysis as 'attenders' (109 at T2, 72 at T3). Those who, despite registration at $\mathrm{T} 1$, reported 'never' attending at T2 were designated 'non-attenders' (297 at T2, 193 at T3). There were no significant differences between CFS attender and non-attender groups on baseline outcome measures or by composition, including sex $\left(\chi^{2}=0.19\right.$, $P=0.66$ ), nationality $\left(\chi^{2}=0.01, P=0.93\right)$, vulnerability designation ( $\chi^{2}=0.55, P=0.46$ ), or formal school attendance at T1 $\left(\chi^{2}=1.51, P=0.22\right.$ ) except for developmental assets where CFS attenders had slightly higher baseline levels as compared to those who did not attend $(P=0.03)$. After primary CFS activities were concluded (between T2 and T3), approximately $59.1 \%(\mathrm{n}=114)$ of the original CFS non-attenders (waitlist control) were offered and participated in programming. This subgroup was removed from further T2-T3 analyses related to CFS attendance efficacy such that 'nonattenders' are defined as never having attended CFS programming.

Bivariate findings offered additional exploration into factors that may be associated with the main longitudinal outcomes. After adjusting for age, gender, nationality, baseline vulnerability, school participation, and $\mathrm{T} 1$ and $\mathrm{T} 2$ outcome scores, results from longitudinal GLM models are reported in Table 2 for one-year predictors of outcome scores and Table 3 for impact of CFS attendance at T2. Intervention attendance rarely predicted primary program outcomes and no Cohen's $d$ estimates were statistically significant $(P<0.05$, Table 3).

\section{PROTECTION CONCERNS}

The type of reported child protection concerns did not change appreciably over time (Figure 2, Plate A), except that by follow-up (T3), school attendance ceased to be a highly reported concern. At the end of the intervention (T2) fewer concerns were reported across the entire sample, as compared to T1, but showed no statistically significant changes within age-group stratified analyses (data not shown). While children aged 10-12 who attended CFS reported fewer concerns than those who did not attend $(\beta=-1.16, P=0.05$, Table 3) immediately after the intervention (T2), CFS attendance, gender, and vulnerability were not predictors of protection concerns at $\mathrm{T} 3$ (Table 2). Across all age ranges at $\mathrm{T} 3$, those who reported caregiver stress related to lost livelihood and being of non-Jordanian nationality (Syrian 
or Palestinian) reported increased protection concerns (nationality: all ages $\beta=-1.65, P<0.001 ; 6-9$ y. $\beta=-2.74$, $P<0.001 ; 10-12$ y. $\beta=-1.66, P<0.001 ; 13-18$ y. $\beta=-0.99$, $P=0.06$; lost livelihoods: all ages $\beta=4.08, P<0.001 ; 6-9 \mathrm{y}$. $\beta=3.42, \quad P<0.001 ; 10-12$ y. $\beta=4.20, \quad P<0.001 ; 13-18$ y. $\beta=4.55, P<0.001$ : Table 2). Children who attended school at both baseline (T1) and follow-up (T3) reported lower levels of protection concerns at follow-up (T3) than those who did not attend school. However, those who attended school at endline (T2) reported more protection concerns at followup (T3) than those who did not attend, especially amongst younger (6-9 year old) children (Table 2 and Online Supplementary Document, Table S1).

\section{CAREGIVER STRESS}

Caregiver stresses remained similar throughout the study period, with the exception that at T3 water had ceased to be a highly reported source of stress (Figure 2, Plate B). CFS attendance, gender, and vulnerability were not predictors of more caregiver stress sources (Table 2). Jordanian nationals reported fewer sources of caregiver stress at followup (T3), as compared to non-Jordanian nationals, across all age groups (all ages $\beta=-2.06, P<0.001 ; 6-9$ y. $\beta=-2.65$, $P<0.001 ; 10-12$ y. $\beta=-1.62, P<0.001 ; 13-18$ y. $\beta=-1.76$, $P<0.001$, Table 2). Those who attended school at baseline (T1) reported fewer caregiver stresses at follow-up (T3) (all ages $\beta=-0.66, P=0.02 ; 6-9$ y. $\beta=-0.75, P=0.11 ; 10-12$ y. $\beta=-0.18, P=0.78 ; 13-18$ y. $\beta=-0.8, P=0.32$, Table 2 ). 
Table 1. Baseline participant demographics and descriptive characteristics by age

\begin{tabular}{|c|c|c|c|c|c|c|c|c|}
\hline \multirow[b]{2}{*}{ Age (standard deviation) } & \multicolumn{2}{|c|}{$\begin{array}{l}\text { Entire sample } \\
\qquad(n=406)\end{array}$} & \multicolumn{2}{|c|}{$\begin{array}{l}\text { Children 6-9 } \\
\qquad(n=165)\end{array}$} & \multicolumn{2}{|c|}{$\begin{array}{l}\text { Children 10-12 } \\
\qquad(\mathrm{n}=114)\end{array}$} & \multicolumn{2}{|c|}{$\begin{array}{l}\text { Children 13-17 } \\
\qquad(\mathrm{n}=127)\end{array}$} \\
\hline & 10.7 & 3.1 & 7.6 & 1.1 & 11 & 0.9 & 14.4 & 1.3 \\
\hline \multicolumn{9}{|l|}{ Gender (\%) } \\
\hline Female & 220 & $54.2 \%$ & 84 & $50.9 \%$ & 59 & $51.8 \%$ & 77 & $60.6 \%$ \\
\hline \multicolumn{9}{|l|}{ Nationality (\%) } \\
\hline Jordanian & 139 & $34.2 \%$ & 50 & $30.3 \%$ & 48 & $42.1 \%$ & 41 & $32.3 \%$ \\
\hline Syrian & 241 & $59.4 \%$ & 110 & $66.7 \%$ & 62 & $54.4 \%$ & 69 & $54.3 \%$ \\
\hline Palestinian & 24 & $5.9 \%$ & 5 & $3.0 \%$ & 5 & $4.4 \%$ & 14 & $11.0 \%$ \\
\hline \multicolumn{9}{|l|}{ Vulnerability designation (\%) } \\
\hline Yes & 49 & $12.1 \%$ & 27 & $16.4 \%$ & 8 & $7.0 \%$ & 14 & $11.0 \%$ \\
\hline \multicolumn{9}{|c|}{ Formal school attendance (\%) } \\
\hline Yes & 308 & $75.9 \%$ & 129 & $78.2 \%$ & 95 & $83.3 \%$ & 84 & $66.1 \%$ \\
\hline \multicolumn{9}{|l|}{ Extracurricular activities (\%) } \\
\hline Always & 19 & $4.7 \%$ & 6 & $3.7 \%$ & 5 & $4.4 \%$ & 8 & $6.3 \%$ \\
\hline Sometimes & 38 & $9.4 \%$ & 9 & $5.5 \%$ & 14 & $12.3 \%$ & 15 & $11.8 \%$ \\
\hline Never & 348 & $85.9 \%$ & 149 & $90.9 \%$ & 95 & $83.3 \%$ & 104 & $81.9 \%$ \\
\hline \multicolumn{9}{|c|}{ Primary study Measures (standard deviation) } \\
\hline Protection concerns & 3.1 & 2.9 & 3.7 & 3.3 & 2.4 & 2.4 & 2.9 & 2.8 \\
\hline Caregiver stress & 2.2 & 2.1 & 2.6 & 2.2 & 1.8 & 1.9 & 1.9 & 2.0 \\
\hline Mental health & 37.7 & 8.6 & 38.9 & 8.8 & 35.5 & 6.3 & 38.1 & 8.8 \\
\hline Development assets & 26.5 & 6.8 & 25.5 & 6.4 & 27.6 & 6.2 & 25.5 & 8.2 \\
\hline
\end{tabular}


Table 2. Primary one-year predictors of child protection concerns, caregiver stress, mental health, and

developmental assets by age

\begin{tabular}{|c|c|c|c|c|}
\hline & Complete Sample & Children 6-9 & Children 10-12 & Children 13-17 \\
\hline & $\beta(95 \% \mathrm{Cl})$ & $\beta(95 \% \mathrm{Cl})$ & $\boldsymbol{\beta}(95 \% \mathrm{Cl})$ & $\beta(95 \% \mathrm{Cl})$ \\
\hline Protection concerns & $\mathrm{n}=277$ & $\mathrm{n}=117$ & $n=81$ & $n=79$ \\
\hline Age & $-0.01(-0.12-0.05)$ & $-0.02(-0.41-0.37)$ & $0.67(0.18-1.15)^{* *}$ & $0.14(-0.20-0.48)$ \\
\hline Gender, (ref: girls) & $0.01(-0.48-0.49)$ & $-0.31(-1.10-0.48)$ & $0.72(-0.17-1.61)$ & $0.20(-0.67-1.07)$ \\
\hline Nationality (ref: non-Jordanian) & $-1.65(-2.29--1.02)^{* * *}$ & $-2.74(-4.04--1.44)^{* * *}$ & $-1.66(-2.64--0.69)^{* * *}$ & $-0.99(-2.04-0.05)$ \\
\hline Attended school, baseline & $-1.05(-1.87--0.23)^{*}$ & $-1.68(-3.17--0.18)^{*}$ & - & - \\
\hline Attended school, follow-up & $-0.90(-1.79--0.01)^{*}$ & - & - & - \\
\hline Lost livelihood, follow-up & $4.08(3.49-4.67)^{* * *}$ & $3.42(2.41-4.43)^{* * *}$ & $4.20(3.11-5.28)^{* * *}$ & $4.55(3.41-5.70)^{* * *}$ \\
\hline Caregiver stress & $\mathrm{n}=328$ & $n=168$ & $n=81$ & $n=79$ \\
\hline Age & $-0.06(-0.14-0.02)$ & $-0.07(-0.40-0.27)$ & $0.49(0.02-0.96)^{*}$ & $0.06(-0.25-0.38)$ \\
\hline Gender, (ref: girls) & $-0.06(-0.51-0.40)$ & $-0.38(-1.09-0.34)$ & $0.37(-0.49-1.22)$ & $0.06(-0.77-0.83)$ \\
\hline Nationality (ref: non-Jordanian) & $-2.06(-2.61--1.50)^{* * *}$ & $-2.65(-3.59--1.71)^{* * *}$ & $-1.62(-2.51--0.72)^{* * *}$ & $-1.76(-2.68--0.83)^{* * *}$ \\
\hline Attended school, baseline & $-0.66(-1.22--0.09)^{*}$ & $-0.75(-1.67--0.17)$ & $-0.18(-1.44-1.08)$ & $-0.48(-1.43-0.46)$ \\
\hline Mental mealth & $\mathrm{n}=201$ & $\mathrm{n}=92$ & $n=58$ & $n=51$ \\
\hline Age & $1.23(0.59-1.87)^{* * *}$ & $-0.79(-4.06-2.48)$ & $-1.17(-3.52-1.18)$ & $-0.01(-1.66-1.63)$ \\
\hline Gender, (ref: girls) & $-2.22(-6.01-1.56)$ & $0.29(-6.19-9.81)$ & $-1.05(-5.31-3.20)$ & $-5.98(-9.96--2.01)^{* *}$ \\
\hline Nationality (ref: non-Jordanian) & $-0.70(-4.77-3.38)$ & $-1.81(-6.19-9.81)$ & $-4.63(-8.90--0.35)^{*}$ & $-2.78(-7.29-1.73)$ \\
\hline Lost livelihood, follow-up & - & - & - & $-7.59(-12.75--2.44)^{* *}$ \\
\hline Developmental assets & $\mathrm{n}=158$ & $\mathrm{n}=92$ & $n=66$ & $n=49$ \\
\hline Age & $0.20(-0.18-0.57)$ & $-0.56(-1.85-0.74)$ & $0.90(-0.45-2.26)$ & $0.29(-0.87-1.45)$ \\
\hline Gender, (ref: girls) & $-1.05(-2.09-0.81)$ & $-0.37(-2.61-3.34)$ & $-0.79(-3.29-1.70)$ & $-1.20(-4.19-1.80)$ \\
\hline Nationality (ref: non-Jordanian) & $1.43(-0.70-3.56)$ & $3.35(-0.10-6.81)$ & $0.43(-2.04-2.91)$ & $-0.99(-4.57-2.59)$ \\
\hline Vulnerable, follow-up & - & $-6.84(-12.61--1.07)^{*}$ & - & - \\
\hline Attended school, endline & $-0.50(-3.58-2.59)$ & - & $9.07(3.38-14.75)^{* *}$ & $0.71(-2.87-4.30)$ \\
\hline Attended school, follow-up & $3.84(0.65-7.02)^{*}$ & - & $-2.70(-8.81-3.41)$ & $-0.54(-4.55-3.47)$ \\
\hline Mental Health, follow-up & - & $0.52(0.43-0.61)^{* * *}$ & - & - \\
\hline Caregiver stress, baseline & $-0.83(-1.49--0.18)^{*}$ & $-0.71(-1.43--0.02)$ & - & - \\
\hline
\end{tabular}

Notes: ${ }^{<}<0.05 ; * * *^{*} 0.01 ; * * * * 00.001$. Bold type indicates statistical significance $P<0.05$. - indicates variable was not included in final adjusted model. 
Table 3. Four-month impact of intervention attendance on protection concerns, caregiver stress,

mental health, and developmental assets by age

\begin{tabular}{|c|c|c|c|c|c|c|c|c|}
\hline & \multicolumn{2}{|c|}{ Complete sample } & \multicolumn{2}{|c|}{ Children 6-9 } & \multicolumn{2}{|c|}{ Children 10-12 } & \multicolumn{2}{|c|}{ Children 13-17 } \\
\hline & $\beta(95 \% \mathrm{Cl})$ & Cohen's $d$ & $\beta(95 \% \mathrm{Cl})$ & Cohen's $d$ & $\beta(95 \% \mathrm{Cl})$ & Cohen's $d$ & $\boldsymbol{\beta}(95 \% \mathrm{Cl})$ & Cohen's $d$ \\
\hline Protection concerns & $-0.48(-1.13-0.17)$ & 0.09 & $-0.72(-1.83-0.38)$ & 0.24 & $-1.16(-2.32-0.00)$ & 0.27 & $0.23(-0.95-1.40)$ & -0.19 \\
\hline Caregiver stress & $-0.19(-0.62-0.25)$ & 0.00 & $-0.26(-1.01-0.50)$ & 0.04 & $-0.66(-1.46-0.14)$ & 0.20 & $0.25(-0.49-0.98)$ & -0.18 \\
\hline Mental health & $-1.36(-3.48-0.77)$ & 0.18 & $-3.37(-7.10-0.36)$ & 0.48 & $-2.16(-5.57-1.26)$ & 0.20 & $1.88(-2.09-5.86)$ & -0.12 \\
\hline Development assets & $1.69(0.24-3.14)$ & 0.07 & $1.87(-0.77-4.50)$ & 0.03 & $0.78(-1.76-3.33)$ & 0.14 & $1.12(-1.31-3.54)$ & 0.05 \\
\hline
\end{tabular}

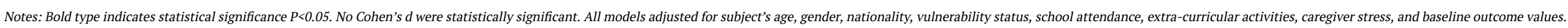


Plate A

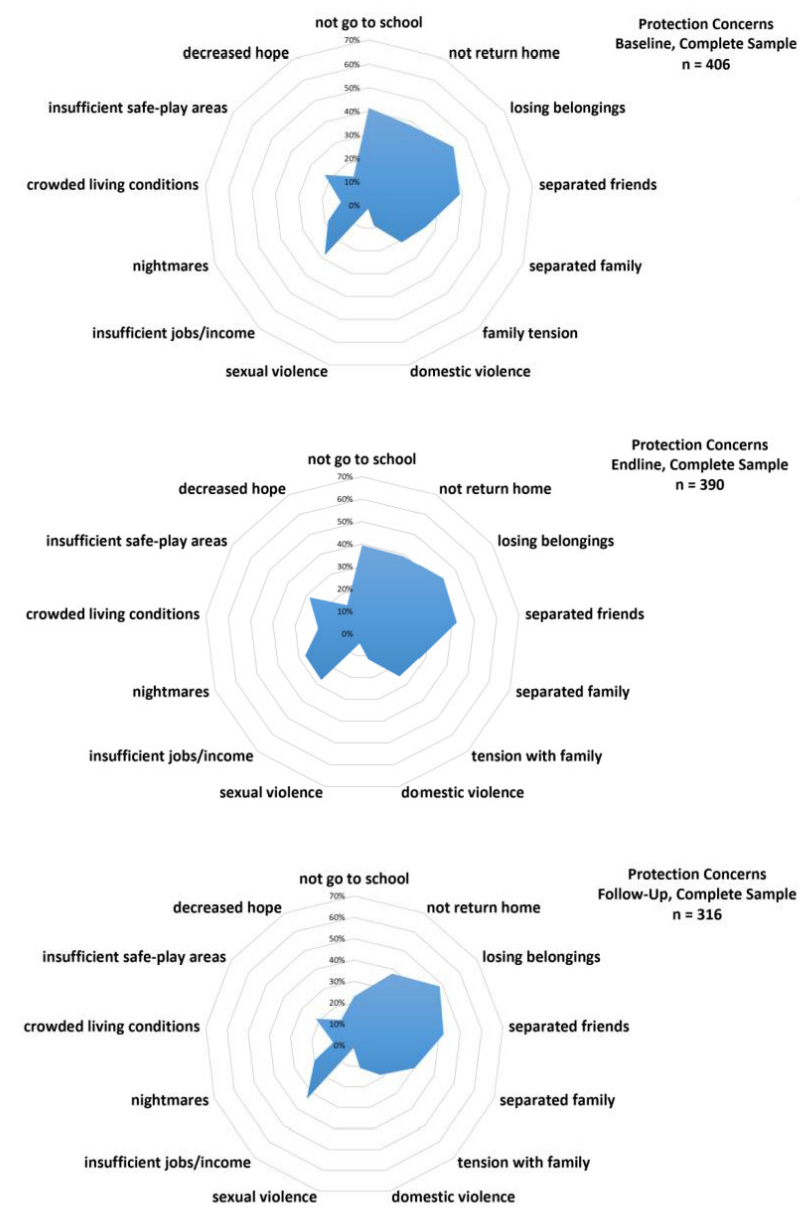

Plate B

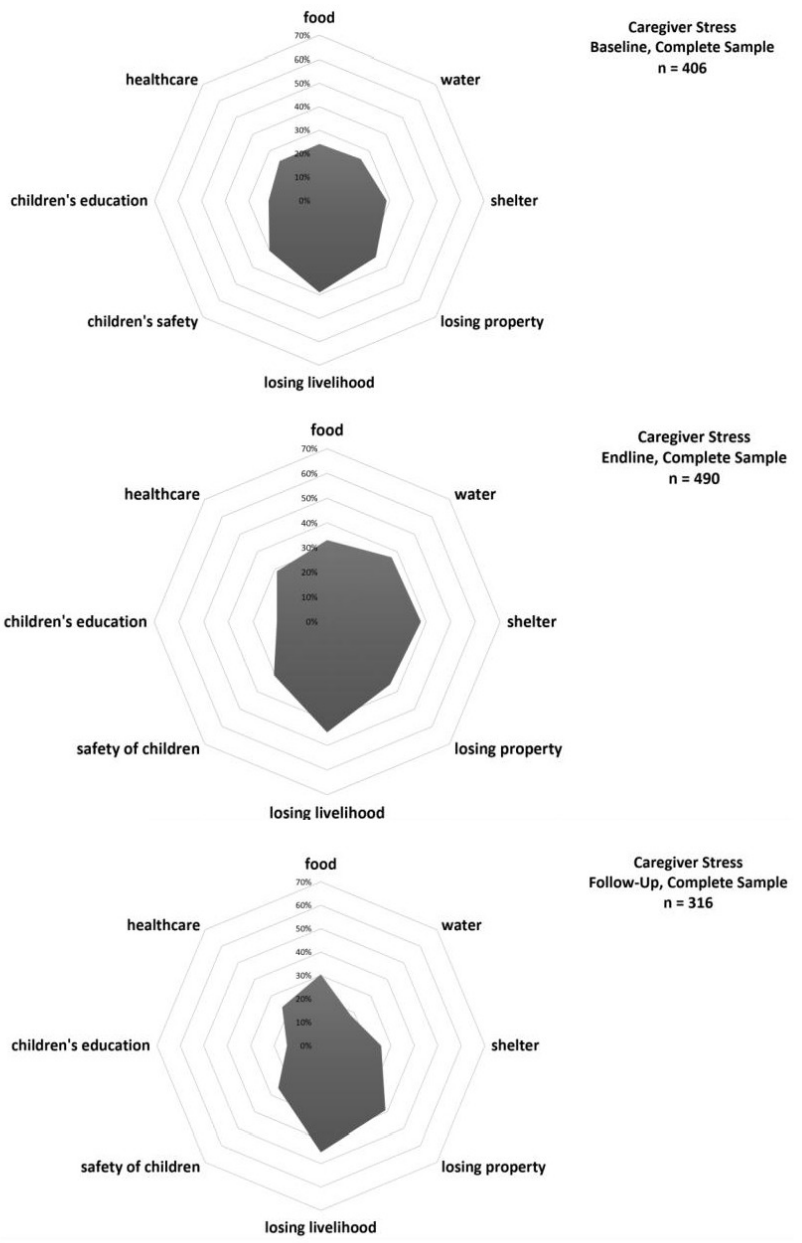

Figure 2. Spider diagrams. A. Protection concerns. B. Caregiver stresses.

\section{MENTAL HEALTH}

Overall, mental health symptoms consistent with the Diagnostic and Statistical Manual of Mental Disorders - IV criteria for depression and anxiety remained below clinical levels and decreased across the study period (Figure 3 ). Amongst older children only, boys reported fewer symptoms than girls of the same age (T3) (13-18 y. $\beta=-5.98$, $P=0.003$, Table 2). Only Jordanian 10 to 12 year old children reported fewer symptoms as compared to non-Jordanian nationals $(10-12$ y. $\beta=-4.63, p=0.03$, Table 2$)$. Older children reporting the stress related to lost livelihoods at follow-up (T3) reported lower levels of symptoms (13-18 y. $\beta=-6.97, P<0.001$, Table 2) as compared to those reporting higher stress related to lost livelihoods. CFS attendance, vulnerability, and school attendance were not predictors of mental health symptoms over time.

\section{DEVELOPMENTAL ASSETS}

Overall, developmental assets declined across the study period (Figure 3). Vulnerability (except for younger children), gender, and nationality were not significant predictors of developmental assets over time. Amongst young children, those who reported higher vulnerability at follow-up (T3) reported lower levels of developmental assets at follow-up (T3) (6-9 y. vulnerability: $\beta=-6.84, P=0.02$; Table 2). Young children who had higher levels of depression and anxiety at follow-up (T3) also reported slightly higher developmental assets at follow-up (T3) (6-9 y. $\beta=0.52, P<0.001)$. Children aged 10 to 12 years attending school at endline (T2) reported, on average, more developmental assets than those children of the same age not attending school (10-12 y. $\beta=9.07, P=0.002$, Table 2). Children who attended CFS reported more assets at baseline (T1) and endline (T2) than those who did not attend ( $\beta=1.69, P=0.02$, Table 3 ); however, this finding did not hold through age subgroup analyses, or at follow-up (T3).

\section{NATIONALITY}

We explored the role of nationality on primary study outcomes across all analyses (Online Supplementary Document, Table S3). Jordanian children, as compared to nonJordanian children, had better baseline levels of primary outcomes (protection concerns, caregiver stress, mental 


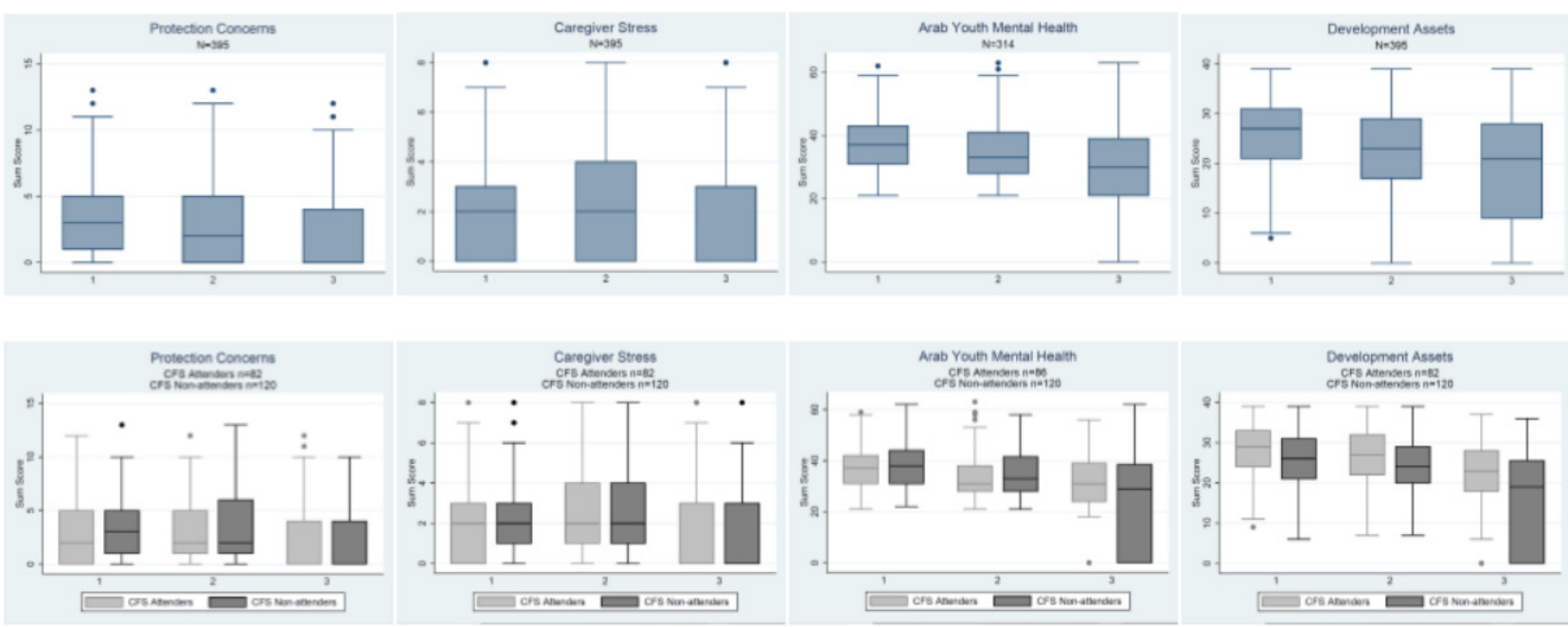

Figure 3. Trends in protection concerns, caregiver stress, mental health symptoms, and developmental assets over time for all children and by intervention attendance.

Notes. Trends across time for Arab Youth Mental Health - in both complete sample and in sample stratified by attendance - were statistically significant $(P<0.05)$. Time periods 1,2 , and 3 correspond to baseline, endline (three months post-baseline, intervention end), and follow-up (one year post-baseline).

health, and developmental assets). In all subsequent analyses, Jordanian children, as compared to non-Jordanian children, continued to do better on most outcomes (Tables 2, S1, S2, available online). Jordanian children, however, were not more likely to participate in the intervention than nonJordanian children (Online Supplementary Document, Table S3).

\section{SCHOOL ATTENDANCE}

We conducted additional sub-analyses into the impact of school attendance on primary outcomes. Over the study period school attendance increased, with $75.9 \%(n=308)$ attending at baseline (T1), 77.6\% $(\mathrm{n}=326)$ at endline $(\mathrm{T} 2$, nonstatistically significant change), and $86.7 \%(n=261)$ at follow-up (T3, $P<0.001$ change, data not shown). There were no statistically significant differences in school attendance between those who attended CFS and those who did not - both across the entire sample and within subgroup age analyses (Table 1, S4, available online) - nor did school attendance predict CFS attendance. Those who attended school at baseline (T1) reported fewer child protection concerns (complete $\beta=-1.33, P=0.02 ; 6-9$ y. $\beta=-2.71, P=0.01$, Online Supplementary Document, Table S1) and caregiver stress (complete $\beta=-1.37, \quad P=0.002 ; 6-9$ y. $\beta=-2.50$, $P=0.003$, Online Supplementary Document, Table S1) at follow-up (T3) than those who did not. Those who attended school at follow-up (T3) reported higher developmental assets (complete $\beta=5.08, P<0.001 ; 13-17$ y. $\beta=5.56, P<0.001$, Online Supplementary Document, Table S1) at follow-up (T3) as compared to those who did not attend school at follow-up (T3). As in other models, Jordanian children, as compared to non-Jordanian children, generally had better outcomes at follow-up (T3) (protection concerns complete $\beta=-3.26, \quad P<0.001$; caregiver stress complete $\beta=-2.55$, ${ }^{*} \mathrm{P}<{ }^{*} 0.001$; mental health complete $\beta=-3.80, P=0.005$; developmental assets complete $\beta=2.08, P=0.008$; Online Sup- plementary Document, Table S1). Models exploring participation in extracurricular activities, excluding the primary intervention, did not produce statistically significant results nor change the overall effect of schooling on primary outcomes (data not shown).

\section{LIVELIHOOD LOSS STRESS}

Given the emerging importance of stress related to livelihood loss in predicting the primary study outcomes, we conducted additional sub-analyses to better understand its predictive role on primary outcomes. Reported stress related to loss of livelihood or fear of livelihood loss was the most often endorsed source of caregiver stress (Figure 2, Plate B), remained constant across the study period (T1: 27.7\%; T2: 27.4\%; T3: 33.2\%), did not differ between intervention attenders and non-attenders at baseline (T1, Online Supplementary Document, Table S4), and did not predict intervention attendance. Those who endorsed stress related to livelihood loss (or fear of livelihood loss) at follow-up (T3) had higher levels of child protection concerns (complete $\beta=4.60, P<0.001 ; 6-9$ y. $\beta=4.54, P<0.001 ; 10-12$ y. $\beta=4.61, P<0.001 ; 13-17$ y. $\beta=4.69, P<0.001$, Online Supplementary Document, Table S2). Stress related to livelihood loss (or fear of loss) did not significantly predict developmental assets or mental health symptoms over the study period, save for older children (13-17 y. mental health: T1 stress $\beta=7.09, P=0.002$, T3 stress $\beta=-6.97, P=0.007$; developmental assets: T2 stress $\beta=-3.72, P=0.03$, Online Supplementary Document, Table S2). Again, in these models, Jordanian children, as compared to non-Jordanian children, generally had better outcomes at follow-up (T3) (protection concerns complete $\beta=-1.74, P<0.001$; caregiver stress complete $\beta=-3.05, * \mathrm{P}=* 0.03$; developmental assets complete $\beta=2.55, P=0.004$; Online Supplementary Document, Table $\mathrm{S} 2)$. 


\section{DISCUSSION}

This study found that over the fifteen-month study period, amongst displacement affected children in Jordan, overall child protection concerns and mental health symptoms improved, caregiver stress remained statistically unchanged, and developmental assets deteriorated. Attending child-focused programming in Zarqa, Jordan in the period between $\mathrm{T} 1$ and $\mathrm{T} 2$ did not significantly impact child protection concerns, caregiver stress, or developmental assets at T2 or T3 overall. In contrast, attending school and concerns over lost livelihoods had significant influence on primary study outcomes. Similar long-term mental health trajectories - beyond intervention effects - towards symptom alleviation have been documented in other intervention studies: among Syrian refugee and Jordanian youth, ${ }^{13}$ war-affected youth in Sierra Leone, ${ }^{33}$ and political violence-affected youth in Indonesia. ${ }^{34}$ Observational studies have also documented symptom alleviation overtime, ${ }^{35,36}$ even in formal symptom class trajectory analyses (beyond scope of current study). ${ }^{37}$

Declining developmental assets over the study period underscore the uncertainty faced by displaced and local children and adolescents in Jordan. Unfamiliar surroundings, fractured communal and social support linkages, and poor economic prospects have strained the protective environment for children affected and displaced by the Syrian conflict. ${ }^{38}$ While not statistically significant across all age groups, high familial vulnerability and caregiver stress predicted lower - and school attendance predicted higher developmental assets (Table 2), consistent with an understanding of familial and community resources as key assets for development .39 The overall trajectory for participants of improved mental health but diminished development assets indicates that resilient adjustment may not so much be driven by - but come through the investment and expenditure of - personal and community assets. ${ }^{27}$

Schooling plays an essential role in both promoting resilience and providing a safe environment where children and adolescents are potentially protected from additional potentially traumatic events and stressors. As compared to other samples of displaced Syrian youth in Jordan where approximately half of surveyed individuals could not continue their education, ${ }^{40,41}$ over $75 \%$ of our sample attended school during the study period. Attending school predicted improvements in child protection concerns, caregiver stress, and higher levels of developmental assets (Table S1, available online). Similar protective influences of schooling have been documented among former child soldiers ${ }^{42,43}$ and in low-income contexts outside humanitarian settings. ${ }^{44}$ However, perhaps because of the potential benefits of attending school, pre-adolescent and adolescent children sometimes face rising concerns with heightened awareness of the challenges of attending a new school. School is not immune from the challenges that cut across existing child protection systems. While not documented in this analysis, Syrian children wishing to enroll in school face many of the same barriers as do low-income youth and caregivers looking to access existing child protection systems, with barriers ranging from child labor and exploitation to precarious local conditions. ${ }^{45}$
Economic stability plays an important role in community, family, and individual security. We found that stress associated with livelihood loss, or the fear of livelihood loss, was associated with increased child protection fears and caregiver stress. As the conflict in Syria rages on, displaced children are confronted daily with the realities of living a life in exile where their developmental trajectories and economic opportunity remain uncertain. The fracturing of communal and social mechanisms of support and protection, such as family and friends, have contributed to rising concerns about the safety of children within the displaced environment and the stress response of caregivers with poor employment opportunities. External programming can either support or undermine not only the uncertainty and stress of livelihood securement, but also the complex communal resources and protective mechanisms. ${ }^{23}$

This study suggests that attendance at the programming in Zarqa, Jordan, did not, overall, improve child or adolescent outcomes in the short term. The inability to identify an intervention impact could reflect a lack of a true impact, consistent a recent meta-analysis of psychosocial interventions. ${ }^{46}$ Amongst older (13-17) children, however, boys who attended the intervention reported fewer symptoms of depression and anxiety $(\beta=-5.90, P=0.003)$, as compared to girls, over time. This is consistent with existing literature on impact evaluations that documents heterogeneous impact across participant subgroups, such as age, gender, socioeconomic status, and access to education. ${ }^{47-49}$ Given that girls and boys had similar levels of mental health symptomology and attendance at baseline, the relative improvement of boys, especially older boys, over girls, indicates a gendered programmatic effect.

This study provides meaningful insight into the trajectory of children and adolescents in Jordan. However, there are certain limitations to this study. The study only evaluated one CFS intervention site and was based on a non-randomized assignment to intervention and control. A high percentage of individuals reported having never participated in other non-CFS or school activities (T1: $86.0 \%$; T2: $74.1 \%$; T3: $88.0 \%$ ). Given the importance of other activities ${ }^{50}$ for child and adolescent development, it is important to consider this population makeup when comparing to other program evaluations. Additionally, for one of our primary outcomes, mental health, we had high item-level missingness (35\%). However, item-level missingness analyses detected no patterning to or measured predictors of the missingness. As with all self-report (or caregiver report) studies, issues of social desirability could lead to underreporting of some conditions deemed stigmatized or unacceptable in this community. Every effort was made during the design and implementation of the study to ensure participants felt comfortable and secure in responding to items, including using trained data collectors experienced in sensitive data collection. This study explores children and adolescents currently residing in Jordan, additional information on study participants before displacement, including more accurate data on displacement timing, events would enable a more complete understanding of the impact of displacement on this population,

In conclusion, while programme benefits were marginal, the relatively significant influences of attending school and 
concerns over lost livelihoods among the study population suggests that to improve child and adolescent wellbeing in contexts of displacement, advancements in access to structural determinants of health, such as education and employment, are essential.

\section{ACKNOWLEDGMENTS}

The study protocol was conducted with ethical approval from the Columbia University Medical Center (Reference AAAJ4352) and the Ministry of Social Development for the Hashemite Kingdom of Jordan. The authors would like to thank Faith Cole and Melany (Clare) Morgenstern for their support with copy editing.

\section{FUNDING}

The research was funded by World Vision International, al elrha R2HC award from the Wellcome Trust and DFID, and additional funding from the National Institute of Mental Health (T32MH096724 and R01MH110872). The funding sources had no role in the analysis or interpretation of this study. The views expressed in this article are the authors' own and not an official position of the institution or funder.

\section{AUTHORSHIP CONTRIBUTIONS}

SH: participated in designing the study, conducted statistical analyses, and contributed to interpretation and drafting of manuscript.
JM: participated in designing the study and provided technical support for project coordination, tool development and translation, training on survey technology, data collection strategy, data analysis, interpretation, and drafting the manuscript.

KS: participated in designing the study, provided overall leadership throughout its duration, and contributed to drafting the manuscript.

AA: was lead investigator and responsible for the overall design and leadership of the study as well as supervision of project staff, data analysis, and manuscript development.

All authors read and approved the final manuscript.

\section{COMPETING INTERESTS}

The authors completed the Unified Competing Interest form at www.icmje.org/coi_disclosure.pdf (available upon request from the corresponding author), and declare no conflicts of interest

\section{CORRESPONDENCE TO:}

Sabrina Hermosilla, PhD, MIA, MPH, MS

455 Main Street, Apt 14E

New York, NY, 10044, USA

shermosi@umich.edu

Submitted: August 27, 2020 GMT, Accepted: November 23, 2020 GMT 


\section{REFERENCES}

1. UNHCR. Global trends forced displacement in 2018. In: Geneva, Switzerland: UNHCR; 2019.

2. Panter-Brick C, Dajani R, Eggerman M, Hermosilla $\mathrm{S}$, Sancilio A, Ager A. Insecurity, distress and mental health: experimental and randomized controlled trials of a psychosocial intervention for youth affected by the Syrian crisis. Journal of child psychology and psychiatry, and allied disciplines. 2017. doi:10.1111/jcpp.12832

3. Neria Y, Galea S, Norris FH. Mental Health and Disasters. Cambridge University Press; 2009.

4. Patel V, Flisher AJ, Nikapota A, Malhotra S. Promoting child and adolescent mental health in low and middle income countries. Journal of child psychology and psychiatry. 2008;49(3):313-334.

5. Reed RV, Fazel M, Jones L, Panter-Brick C, Stein A. Mental health of displaced and refugee children resettled in low-income and middle-income countries: Risk and protective factors. The Lancet. 2012;379(9812):250-265. doi:10.1016/S0140-6736(1 1) $60050-0$

6. Javanbakht A, Rosenberg D, Haddad L, Arfken CL. Mental health in Syrian refugee children resettling in the United States: war trauma, migration, and the role of parental stress. J Am Acad Child Adolesc Psychiatry. 2018;57(3):209-211 e202. doi:10.1016/j.ja ac.2018.01.013

7. Elsafti AM, van Berlaer G, Al Safadi M, et al. Children in the Syrian Civil War: the Familial, Educational, and Public Health Impact of Ongoing Violence. Disaster Med Public Health Prep. 2016;10(6):874-882. doi:10.1017/dmp.2016.165

8. Vervliet M, Lammertyn J, Broekaert E, Derluyn I. Longitudinal follow-up of the mental health of unaccompanied refugee minors. European child \& adolescent psychiatry. 2014;23(5):337-346. doi:10.100 7/s00787-013-0463-1

9. UNHCR. Syria regional refugee response interagency information sharing portal. 2017.

10. UNHCR. Global trends forced displacement in 2015. In: Geneva, Switzerland: UNHCR; 2016.

11. UNHCR. Submission by the UNHCR for the Office of the High Commissioner for Human Rights' compilation report - Universal Periodic Review: Jordan. 2013.
12. Kadir A, Shenoda S, Goldhagen J. Effects of armed conflict on child health and development: A systematic review. PLoS One. 2019;14(1):e0210071. $\underline{\mathrm{d}}$ oi:10.1371/journal.pone.0210071

13. Panter - Brick C, Dajani R, Eggerman M, Hermosilla S, Sancilio A, Ager A. Insecurity, distress and mental health: experimental and randomized controlled trials of a psychosocial intervention for youth affected by the Syrian crisis. Journal of child psychology and psychiatry, and allied disciplines. 2017. doi:10.1111/jcpp.12832

14. Tol W, Song S, Jordans M. Annual Research Review: Resilience and mental health in children and adolescents living in areas of armed conflict - a systematic review of findings in low- and middleincome countries. Journal of Child Psychology and Psychiatry. 2013;54(4):445-460.

15. Kinzie JD, Sack WH, Angell RH, Manson S, Rath B. The psychiatric effects of massive trauma on Cambodian children: I. The children. Journal of the American Academy of Child Psychiatry. 1986;25(3):370-376.

16. Patel V. Why mental health matters to global health. Transcultural psychiatry. 2014;51(6):777-789. doi:10.1177/1363461514524473

17. Whiteford HA, Ferrari AJ, Degenhardt L, Feigin V, Vos T. The global burden of mental, neurological and substance use disorders: an analysis from the Global Burden of Disease Study 2010. PLoS One. 2015;10(2):e0116820. doi:10.1371/journal.pone.0116 $\underline{820}$

18. Alfano CA, Lau S, Balderas J, Bunnell BE, Beidel DC. The impact of military deployment on children: Placing developmental risk in context. Clin Psychol Rev. 2016;43:17-29. doi:10.1016/j.cpr.2015.11.003

19. Gartland D, Riggs E, Muyeen S, et al. What factors are associated with resilient outcomes in children exposed to social adversity? A systematic review. BMJ Open. 2019;9(4):e024870. doi:10.1136/bmjopen-201 $\underline{8-024870}$

20. Betancourt TS, Thomson DL, Brennan RT, Antonaccio CM, Gilman SE, VanderWeele TJ. Stigma and acceptance of Sierra Leone's child soldiers: a prospective longitudinal study of adult mental health and social functioning. J Am Acad Child Adolesc Psychiatry. 2019. doi:10.1016/i.jaac.2019.05.026 
21. Rutter M. Poverty and child mental health: natural experiments and social causation. JAMA. 2003;290(15):2063-2064. doi:10.1001/jama.290.15.20 $\underline{63}$

22. Masten AS, Narayan AJ. Child development in the context of disaster, war, and terrorism: pathways of risk and resilience. Annu Rev Psychol.

2012;63:227-257. doi:10.1146/annurev-psych-12071 $\underline{0-100356}$

23. Viner RM, Ozer EM, Denny S, et al. Adolescence and the social determinants of health. Lancet. 2012;379(9826):1641-1652. doi:10.1016/S0140-673 6(12)60149-4

\section{UNICEF. No lost generation.}

25. Ager A, Annan J, Panter-Brick C. Resilience: From conceptualization to effective intervention. Policy Brief for IRC Strategic Planning Meeting, May 6, 2013. 2013.

26. Ungar M, Ghazinour M, Richter J. Annual research review: What is resilience within the social ecology of human development? Journal of Child Psychology and Psychiatry and Allied Disciplines. 2013;54(4):348-366. doi:10.1111/jcpp.12025

27. Ager A, Metzler J. Where there is no intervention: insights into processes of resilience supporting waraffected children. Peace and Conflict: Journal of Peace Psychology. 2017;23(1):67-75.

28. Metzler J, Ishaq M, Hermosilla S, Mumba E, Ager A. Evaluation of child friendly spaces: Jordan field study report: a CFS implemented by world vision and partners in Zarqa, Jordan. New York City \& Amman: Columbia University \& World Vision. 2015.

29. Makhoul J, Nakkash R, El Hajj T, et al. Development and validation of the Arab Youth Mental Health Scale. Community Mental Health Journal. 2011;47:331-340.

30. Child Protection Working Group. Child protection rapid assessment toolkit. 2012.

31. Scales P, Roehlkepartain E, Wallace T, Inselman A, Stephenson P, Rodriguez M. Brief report: Assessing youth well-being in global emergency settings: Early results from the Emergency Developmental Assets Profile. Journal of Adolescence. 2015;45:98-102.

32. Cohen J. Statistical Power Analysis for the Behavioral Sciences. Routledge; 1977.
33. Betancourt TS, McBain R, Newnham EA, et al. A behavioral intervention for war-affected youth in Sierra Leone: a randomized controlled trial. Journal Of The American Academy Of Child And Adolescent Psychiatry. 2014;53(12):1288-1297. doi:10.1016/j.jaa c.2014.09.011

34. Tol WA, Komproe IH, Susanty D, Jordans MJD, Macy RD, De Jong JTVM. School-based mental health intervention for children affected by political violence in Indonesia: a cluster randomized trial. JAMA. 2008;300(6):655-662. doi:10.1001/jama.300.6.655

35. Keles S, Friborg O, Idsøe T, Sirin S, Oppedal B. Resilience and acculturation among unaccompanied refugee minors. International Journal of Behavioral Development. 2018;42(1):52-63.

36. Hassan G, Ventevogel P, Jefee-Bahloul H, BarkilOteo A, Kirmayer L. Mental health and psychosocial wellbeing of Syrians affected by armed conflict. Epidemiology and psychiatric sciences. 2016;25(2):129-141.

37. Tay AK, Jayasuriya R, Jayasuriya D, Silove D.

Twelve-month trajectories of depressive and anxiety symptoms and associations with traumatic exposure and ongoing adversities: a latent trajectory analysis of a community cohort exposed to severe conflict in Sri Lanka. Transl Psychiatry. 2017;7(8):e1200. doi:1 $\underline{0.1038 / \text { tp.2017.166 }}$

38. UNICEF. UNICEF Assesment Shows 85 per Cent of Syrian Refugee Children in Host Communities Live in Poverty. UNICEF; 2018.

39. Benson PL, Scales PC, Syvertsen AK. The contribution of the developmental assets framework to positive youth development theory and practice. In: Advances in Child Development and Behavior. Vol 41. Elsevier; 2011:197-230.

40. UNHCR. Syria Crisis: Education interrupted. 2013.

41. UNICEF. UNICEF infographic: Syria's children under siege. 2014.

42. Kohrt BA, Jordans MJ, Tol WA, et al. Social ecology of child soldiers: child, family, and community determinants of mental health, psychosocial wellbeing, and reintegration in Nepal. Transcult Psychiatry. 2010;47(5):727-753. doi:10.1177/1363461 $\underline{510381290}$

43. Betancourt TS, Borisova I, Williams TP, et al. Psychosocial adjustment and mental health in former child soldiers--systematic review of the literature and recommendations for future research. J Child Psychol Psychiatry. 2013;54(1):17-36. doi:10.1111/j.1469-761 0.2012.02620.x 
44. O’Donnell DA, Roberts WC, Schwab-Stone ME. Community violence exposure and post-traumatic stress reactions among Gambian youth: the moderating role of positive school climate. Soc Psychiatry Psychiatr Epidemiol. 2011;46(1):59-67. do i:10.1007/s00127-009-0162-x

45. No Lost Generation. We Made a Promise: Ensuring Learning Pathways and Protection for Syrian Children and Youth. No Lost Generation; 2018.

46. Purgato M, Gross AL, Betancourt T, et al. Focused psychosocial interventions for children in lowresource humanitarian settings: a systematic review and individual participant data meta-analysis. Lancet Glob Health. 2018;6(4):e390-e400. doi:10.1016/S221 4-109X(18)30046-9

47. Annan J, Sim A, Puffer ES, Salhi C, Betancourt TS. Improving mental health outcomes of burmese migrant and displaced children in Thailand: a community-based randomized controlled trial of a parenting and family skills intervention. Prev Sci. 2017;18(7):793-803. doi:10.1007/s11121-016-0728-2
48. Bangpan M, Lambert F, Chiumento A, Dickson K. The impact of mental health and psychosocial support programmes for populations affected by humanitarian emergencies: a systematic review protocol. 2016.

49. Fazel M, Patel V, Thomas S, Tol W. Mental health interventions in schools in low-income and middleincome countries. Lancet Psychiatry. 2014;1(5):388-398. doi:10.1016/S2215-0366(14)7035 $\underline{7-8}$

50. Durlak JA, DuPre EP. Implementation matters: a review of research on the influence of implementation on program outcomes and the factors affecting implementation. Am J Community Psychol. 2008;41(3-4):327-350. doi:10.1007/s10464-0 08-9165-0 


\section{SUPPLEMENTARY MATERIALS}

\section{Online Supplementary Document}

Download: https://www.joghr.org/article/18233-resilience-and-adjustment-trajectories-amongst-children-indisplacement-affected-communities-in-zarqa-jordan/attachment/48259.docx 